\title{
Robustness of general Triple I method for fuzzy soft sets
}

\author{
Lu Wang ${ }^{1}$ Keyun Qin ${ }^{2}$, \\ ${ }^{1}$ College of Mathematics, Southwest Jiaotong University, \\ Chengdu, 610031, Sichuan, PR China ${ }^{\dagger}$ \\ E-mail:965151752@qq.com \\ ${ }^{2}$ E-mail: keyunqin@263.net
}

Received 9 March 2018

Accepted 18 May 2018

\begin{abstract}
The stability and robustness analysis is a vital issue of fuzzy soft inference. In this paper, $\lambda$-Triple I inference methods based on the fuzzy soft modus ponens (FSMP) and fuzzy soft modus tollens (FSMT) are presented. The related computational formulas for inference conclusions with respect to the residual pair are given. Robustness of $\lambda$-Triple I inference methods for FSMP and FSMT are analyzed. Finally, robustness analysis of general Triple I inference methods for multiple fuzzy soft rule in the FSMP model are presented.
\end{abstract}

Keywords: Fuzzy soft set, $\lambda$ - Triple I method, robustness, Left continuous t-norm

\section{Introduction}

As a result of the Composition Rule of Inference $(\mathrm{CRI})^{1}$ proposed by Zadeh, multifarious fuzzy reasoning method have been proposed ${ }^{2,3,4}$. Robustness is an important evaluation criteria for fuzzy inference. In fuzzy environment, robustness is used to study how the error in the premises affects consequents of fuzzy inference. The robustness analysis is usually based on particular proximity measure of fuzzy set ${ }^{5}$. Dai et al. studied perturbations based on the logical equivalence measure for fuzzy sets ${ }^{6}$. On the basis of the $\delta$ - equalities ${ }^{7}$, the robustness of CRI is analyzed. The robustness analysis which is based on measuring the errors of conclusions is generated by the deviations of presuppositions in fuzzy inference $\delta$ - equalities. Li discussed the robustness of fuzzy inference by analysis for perturbation of membership functions and proposed a way for distinguishing different categories of fuzzy connectives whether are the most robust elements $^{8}$. Then, Li also discussed the robustness for fuzzy illation based on divergence measures ${ }^{9}$.

Molodtsov firstly presented the soft set theory in $1999^{10,11}$. It has been proven that the soft set theory has a great potential for application in a lot of fields such as texture classification, analysis of the data and decision making ${ }^{12,13,14}$. The researches of soft set theory make rapid progress. Maji et al. and Ali et al. defined a few operations of soft sets ${ }^{15}$ and made a theoretical study on the soft set theory ${ }^{16}$. Maji et al. discussed the mixed structures with respect to soft sets and fuzzy sets ${ }^{17}$. M. Khan introduced the hybrid structure involving soft set and complex number representation of intuitionistic fuzzy set and presented some basic theoretic operations on this

\footnotetext{
* E-mail: keyunqin@263.net(Keyun Qin)

† E-mail: 965151752@qq.com(Lu Wang),
} 
hybrid structure such as union, intersection, restricted union and restricted intersection ${ }^{18}$. S. Bouznad presented a new fusion scheme for ensemble classifiers based on a new concept called Generalized Fuzzy Soft Set (GFSS) ${ }^{19}$. Then, Qin presented an approach to fuzzy soft inference method with respected to fuzzy soft implication operators ${ }^{20}$. Wang proposed the similarity-based approximate reasoning methods for fuzzy soft set and presented the computational formulas for these methods ${ }^{21}$. Xue introduced triple I inference method for intervalvalued fuzzy soft sets and studied the reversibility property of triple I methods of interval-valued fuzzy soft modus ponens (IVFSMP) and interval-valued fuzzy soft modus tollens (IVFSMT) ${ }^{22}$. Xue studied the reversibility and the continuity of fuzzy soft set based triple I reasoning method ${ }^{23}$. Some properties of triple I inference for fuzzy soft sets have been investigated. So far, there has been some progress concerning robustness analysis in fuzzy reasoning. $\mathrm{Jin}^{24}$ proposed the robustness of fuzzy reasoning and gave some robustness results for various fuzzy logic connectives, fuzzy implication operators, inference rules and fuzzy reasoning machines. $\mathrm{Luo}^{25}$ presented the robustness of triple I algorithms based on interval-valued fuzzy sets for fuzzy inference. R. Zanotelli ${ }^{26}$ introduced the robustness analysis of intuitionistic fuzzy difference operators based on the evaluation of the $\delta$ sensitivity in representable fuzzy negations, triangular norms and conorms. However, no one has studied robustness analysis for Triple I fuzzy soft inference and general Triple I fuzzy soft inference yet. In this paper we introduce general Triple I fuzzy soft inference method $(\lambda$-Triple I fuzzy soft inference method) and study robustness analysis of general fuzzy soft inference. Section 2 reviews certain basic definitions and lemmas. Section 3 introduces the $\lambda$ - Triple I fuzzy soft inference. Section 4 discusses robustness analysis of general Triple I fuzzy soft inference based on FSMP and FSMT. In section 5, robustness analysis of general Triple I inference methods for multiple fuzzy soft rule in the FSMP model are discussed. We conclude in section 6 .

\section{Preliminaries}

A fuzzy implication operator is a binary operation $R:[0,1]^{2} \rightarrow[0,1]$ satisfying conditions ${ }^{27}$ :

(1) $R(0,1)=R(1,1)=1, R(1,0)=0$.

(2) $x \leqslant y$ implies $R(z, x) \leqslant R(z, y)$ and $R(y, z) \leqslant$ $R(x, z)$.

Triangular norms (t-norms) are closely related to fuzzy implication operators. A function $T:[0,1]^{2} \rightarrow$ $[0,1]$ is said to be t-norm ${ }^{28}$ if the function $T$ is associative, commutative and satisfy $T(x, 1)=x$ and $x \leqslant y$ implies $T(x, z)=T(y, z)$ for every $x, y, z \in$ $[0,1]$. A t-norm $T$ is called left-continuous t-norm ${ }^{28}$ if $T\left(x, \vee_{i \in I} y_{i}\right)=\underset{i \in I}{\vee} T\left(x, y_{i}\right)$ holds for any $x, y_{i} \in[0,1]$ and $i \in I(I=1,2, \cdots)$. If a t-norm $T$ is associative, we think $T(a, b, c)$ is equal to $T(T(a, b), c)^{28}$.

Definition 1. ${ }^{29}$ For a t-norm $T$ and a fuzzy implication operator $R,(T, R)$ is called a residual pair if

$$
a \leqslant R(b, c) \Leftrightarrow T(a, b) \leqslant c
$$

for every $a, b, c \in[0,1]$.

Theorem 1. ${ }^{27}$ Assume that $T$ is a left continuous $t$-norm, define $R:[0,1]^{2} \rightarrow[0,1]$ as

$$
R(a, b)=\sup \{x \in[0,1] \mid T(a, x) \leqslant b\}
$$

for every $a, b \in[0,1]$. Then $(T, R)$ is a residual pair satisfying ${ }^{29,30}$ :

(1) $R(a, b)=1$ iff $a \leqslant b$;

(2) $R(1, a)=a$;

(3) $T(R(a, c), R(b, c)) \leqslant R(a, b)$;

(4) $T(a, R(a, b)) \leqslant a \wedge b$; Furthermore, if $T$ is continuous, then $T(a, R(a, b))=a \wedge b$;

(5) $R(T(a, b), c)=R(a, R(b, c))$;

(6) $a \leqslant R(b, c) \Leftrightarrow b \leqslant R(a, c)$.

E.P. Klement and R. Mesiar defined the biresidual ${ }^{28}$ as a binary function on $[0,1]$ in the following way:

Definition 2. ${ }^{28}$ The biresidual related to a leftcontinuous triangular norm $T$ is defined by

$$
\rho(p, q)=p \leftrightarrow q=R(p, q) \wedge R(q, p) .
$$


Biresidual $p \leftrightarrow q$ can be used to characterize the similarity between $p$ and $q$. Now, we are going to recall further properties in terms of the biresidual related to the left-continuous triangular norm.

Lemma 2. ${ }^{29,30,31}$ Assume that $T$ is a left-continuous triangular norm and $\rho$ is the biresidual associated with $T$. Then, for any $x, y, z, o \in[0,1]$ :
(1) $x \leftrightarrow 1=x$;
(2) $x=y \Leftrightarrow x \leftrightarrow y=1$;
(3) $x \leftrightarrow y=y \leftrightarrow x$;
(4) $x \leftrightarrow y \leqslant R(x, 0) \leftrightarrow R(y, 0)$
(5) $T(x \leftrightarrow y, y \leftrightarrow z) \leqslant x \leftrightarrow z$;
(6) $(x \leftrightarrow y) \wedge(z \leftrightarrow o) \leqslant(x \vee z) \leftrightarrow(y \vee o)$;
(7) $T(x \leftrightarrow y, z \leftrightarrow o) \leqslant T(x, z) \leftrightarrow T(y, o)$;
(8) $T(x \leftrightarrow y, z \leftrightarrow o) \leqslant R(x, z) \leftrightarrow R(y, o)$.

Lemma 3. ${ }^{28}$ Assume that two arbitrary functions are $h_{1}, h_{2}: O \rightarrow[0,1], \rho$ is the biresidual and a nonempty set is $O$. Then

$$
\begin{aligned}
& \text { (1) } \underset{o \in O}{\wedge}\left(h_{1}(o) \leftrightarrow h_{2}(o)\right) \leqslant \bigwedge_{o \in O} h_{1}(o) \leftrightarrow \bigwedge_{o \in O} h_{2}(o) ; \\
& \text { (2) } \underset{o \in O}{\wedge}\left(h_{1}(o) \leftrightarrow h_{2}(o)\right) \leqslant \underset{o \in O}{\vee} h_{1}(o) \leftrightarrow \underset{o \in O}{\vee} h_{2}(o) \text {. }
\end{aligned}
$$

Molodtsov presented the theory of soft set for handing uncertainties ${ }^{11}$. Assume that $U$ is an initial universe set and $E$ is parameters on the universal set. $(U, E)$ is called a soft space.

Definition 3. ${ }^{11}$ The pair $(f, S)$ is said to be a soft set over $U$ if $S \subseteq E$ and $f: S \rightarrow P(U)$.

That is to say, soft set in Definition 3 is a parameterized family of subsets of universe set. In general, the parameters of a soft space are fuzzy words or sentences associated with fuzzy words. With this in mind, Maji et al. gave the definition of fuzzy soft set $^{17}$.

Definition 4. ${ }^{17}$ Assume that $S$ is subset of $E$, a pair $(f, S)$ is a fuzzy soft set over $U$ if $f: S \rightarrow F(U)$, where $F(U)$ denote the set of all fuzzy subsets over $U$.

In order to investigate fuzzy soft sets based approximate reasoning, Qin extended the definition of fuzzy implication operator to fuzzy soft implication operator $^{20}$.

Definition 5. ${ }^{20}$ Assume that $(f, S)$ and $(g, T)$ are two fuzzy soft sets over $U$ and $V$ respectively. A fuzzy soft implication operator from $(f, S)$ to $(g, T)$ is a fuzzy soft set $(h, K)$ over $U \times V$, where $K \subseteq S \times T, h: K \rightarrow F(U \times V)$ is given by

$$
H(s, t)(u, v)=F(s)(u) \rightarrow G(t)(v)
$$

for all $(s, t) \in K,(u, v) \in U \times V$, and $\rightarrow$ is a fuzzy implication operator.

Based on fuzzy soft implication operators, Qin presented fuzzy soft inference method ${ }^{20}$. Triple I inference methods for FSMP and FSMT are presented. The related calculation formulas for FSMP and FSMT are studied. FSMP and FSMT can be expressed as follows ${ }^{20}$ :

FSMP: Major premise: $(f, S) \rightarrow(g, T)$ and Minor premise: $\left(f^{*}, S\right)$. Conclusion: $\left(g^{*}, T\right)$;

FSMT: Major premise: $(f, S) \rightarrow(g, T)$ and Minor premise: $\left(g^{*}, T\right)$. Conclusion: $\left(f^{*}, S\right)$.

Where $(f, S),\left(f^{*}, S\right)$ and $(g, T),\left(g^{*}, T\right)$ are fuzzy soft sets over $U$ and $V$ respectively. Based on the FSMP and FSMT, Qin presented Principle of triple I in the following:

Principle of triple I for FSMP ${ }^{20}$ : The conclusion $\left(g^{*}, T\right)$ of FSMP is the smallest fuzzy soft set over $V$ satisfying

$$
R\left(R(f(s)(u), g(t)(v)), R\left(f^{*}(s)(u), g^{*}(t)(v)\right)\right)=1
$$

Principle of triple I for FSMT ${ }^{20}$ : The conclusion $\left(f^{*}, S\right)$ of FSMT is the largest fuzzy soft set over $U$ satisfying

$$
R\left(R(f(s)(u), g(t)(v)), R\left(f^{*}(s)(u), g^{*}(t)(v)\right)\right)=1
$$

Theorem 4. ${ }^{20}$ Assume that the implication operator in FSMP and FSMT is the residual implication $R$ of left-continuous $t$-norm $T$. 
(1) The calculation formula of Triple I inference method for FSMP is expressed as: for each $t \in T, v \in V$,

$$
\begin{gathered}
g^{*}(t)(v)=\underset{s \in S}{\vee} \underset{u \in U}{\vee} T(R(f(s)(u), g(t)(v)), \\
\left.f^{*}(s)(u)\right)
\end{gathered}
$$

(2) The calculation formula of Triple I inference method for FSMT is expressed as: for each $u \in U, s \in S$,

$$
\begin{gathered}
f^{*}(s)(u)=\underset{t \in T}{\wedge} \wedge_{v \in V} R(R(f(s)(u), g(t)(v)), \\
\left.g^{*}(t)(v)\right)
\end{gathered}
$$

\section{3. $\lambda$ - Triple I method for fuzzy soft sets}

Upon all these content, we will extend triple I method for fuzzy soft sets to $\lambda$ - triple I method. In the section, we denote $(f, S),\left(f^{*}, S\right)$ and $(g, T)$, $\left(g^{*}, T\right)$ are fuzzy soft set over $U$ and $V$. At first, we will consider $\lambda$ - triple I method for FSMP. That is to say, for given $\lambda \in[0,1]$, our purpose is to seek the optimal solution satisfying the following:

$\lambda$ - Triple I FSMP principle: $\left(g^{*}, T\right)$ satisfying this principal is the smallest fuzzy soft set over $V$ such that

$R\left(R(f(s)(u), g(t)(v)), R\left(f^{*}(s)(u), g^{*}(t)(v)\right)\right) \geqslant \lambda$.

Theorem 5. ( $\lambda$ - Triple I FSMP method) Suppose that a left-continuous triangular norm is $T$ and the implication operator in FSMP is $R$. Then the fuzzy soft set $\left(g_{\lambda^{*}}, T\right)$ satisfying $\lambda$ - Triple I FSM$P$ principle can be expressed as follows: for each $v \in V, t \in T$,

$$
\begin{gathered}
g_{\lambda}^{*}(t)(v)=\underset{\alpha \in S u \in U}{\vee} T(T(\lambda, R(f(s)(u), g(t)(v))), \\
\left.f^{*}(s)(u)\right) .
\end{gathered}
$$

Proof. Firstly, we prove that $g_{\lambda}{ }^{*}(t)(v)$ defined by (4) satisfying (3). For each $u \in U, s \in S, v \in V, t \in T$, we have

$g_{\lambda}{ }^{*}(t)(v) \geqslant T\left(T(\lambda, R(f(s)(u), g(t)(v))), f^{*}(s)(u)\right)$

By Definition 1, it follows that

$T(\lambda, R(f(s)(u), g(t)(v))) \leqslant R\left(f^{*}(s)(u), g_{\lambda}^{*}(t)(v)\right)$,

$\lambda \leqslant R\left(R(f(s)(u), g(t)(v)), R\left(F^{*}(s)(u), g_{\lambda}^{*}(t)(v)\right)\right)$.

Next, suppose that $(h, T)$ is the fuzzy soft set over $V$ and $(h, T)$ satisfies (3). Then we have

$$
R\left(R(f(s)(u), g(t)(v)), R\left(f^{*}(s)(u), h(t)(v)\right)\right) \geqslant \lambda
$$

for each $u \in U, s \in S, v \in V, t \in T$.

By Theorem 1(5), it follow that

$$
\begin{aligned}
& R\left(T\left(T(\lambda, R(f(s)(u), g(t)(v))), f^{*}(s)(u)\right), h(t)(v)\right) \\
& =R\left(T(\lambda, R(f(s)(u), g(t)(v))), R\left(f^{*}(s)(u), h(t)(v)\right)\right) \\
& =R\left(\lambda, R\left(R(f(s)(u), g(t)(v)), R\left(f^{*}(s)(u), h(t)(v)\right)\right)\right) \\
& =1 .
\end{aligned}
$$

Thus, we can conclude that $T(T(\lambda, R(f(s)(u), g(t)(v)))$, $\left.f^{*}(s)(u)\right) \leqslant h(t)(v)$ and hence

$$
\begin{gathered}
g_{\lambda}^{*}(t)(v)=\underset{s \in S}{\vee} \underset{u \in U}{\vee} T(T(\lambda, R(f(s)(u), g(t)(v))), \\
\left.f^{*}(s)(u)\right) \leqslant h(t)(v) .
\end{gathered}
$$

Corollary 6. Suppose that the implication operator in FSMP is $R_{L}$, where $T_{L}(p, q)=(p+q-1) \vee 0$ and $R_{L}(p, q)=(1-p+q) \wedge 1$. Then the calculation formula of $\lambda$ - Triple I inference of (4) is expressed as follows: for each $t \in T, v \in V$,

$$
g_{\lambda}^{*}(t)(v)=\underset{s \in S}{\vee} \underset{u \in E(s, t, v)}{\vee}\left(\left(\lambda+f^{*}(s)(u)-f(s)(u)\right.\right.
$$




$$
\left.+g(t)(v)-1) \wedge\left(\lambda+f^{*}(s)(u)-1\right)\right),
$$

where $E(s, t, v)=\left\{u \in U ; f^{*}(s)(u)-f(s)(u)+\right.$ $\left.g(t)(v)>1-\lambda, f^{*}(s)(u)>1-\lambda\right\}$.

Corollary 7. Suppose that the implication operator in FSMP is $R_{G}$, where $T_{G}(p, q)=p \wedge q$ and

$$
R_{G}(p, q)= \begin{cases}1, & p \leqslant q \\ q . & p>q\end{cases}
$$

Then the calculation formula of $\lambda$-Triple I inference of (4) is expressed as follows: for each $t \in T$, $v \in V$,

$$
\begin{gathered}
g_{\lambda}^{*}(t)(v)=\underset{s \in S u \in U}{\vee} \vee_{u}\left(\lambda \wedge f^{*}(s)(u) \wedge R_{G}(f(s)(u),\right. \\
g(t)(v))) .
\end{gathered}
$$

Now, we consider $\lambda$-triple I method for FSMT.

$\lambda$ - Triple I FSMT principle: $\left(f^{*}, S\right)$ satisfying this principal is the maximal fuzzy soft set over $U$ such that (3) holds.

Theorem 8. ( $\lambda$ - Triple I FSMT method) Assumed that a left-continuous triangular norm is $T$ and the implication operator in FSMT is $R$. Then the fuzzy soft set $\left(f_{\lambda}{ }^{*}, S\right)$ satisfying $\lambda$ - Triple I FSMT principle can be expressed as follows: for each $s \in S, u \in U$,

$$
\begin{gathered}
f_{\lambda}^{*}(s)(u)=\underset{t \in T}{\wedge} \underset{v \in V}{\wedge} R(\lambda, R(R(f(s)(u), g(t)(v)), \\
\left.\left.g^{*}(t)(v)\right)\right) .
\end{gathered}
$$

Proof. Firstly, we prove that $\left(f_{\lambda}{ }^{*}, S\right)$ defined by (5) satisfying (3). For each $u \in$ $U, s \in S, \quad v \in V, t \in T$, we have $f_{\lambda}{ }^{*}(s)(u) \leqslant$ $R\left(\lambda, R\left(R(f(s)(u), g(t)(v)), g^{*}(t)(v)\right)\right)$ and hence $R\left(\lambda, R\left(R(f(s)(u), g(t)(v)), R\left(f_{\lambda}^{*}(s)(u), g^{*}(t)(v)\right)\right)\right)$

$$
\begin{aligned}
& =R\left(\lambda, R\left(f_{\lambda}^{*}(s)(u), R\left(R(f(s)(u), g(t)(v)), g^{*}(t)(v)\right)\right)\right) \\
& =R\left(f_{\lambda}^{*}(s)(u), R\left(\lambda, R\left(R(f(s)(u), g(t)(v)), g^{*}(t)(v)\right)\right)\right) \\
& =1 .
\end{aligned}
$$

Thus, we can conclude that

$$
\lambda \leqslant R\left(R(f(s)(u), g(t)(v)), R\left(f_{\lambda}{ }^{*}(s)(u), g^{*}(t)(v)\right)\right) .
$$

Next, suppose that $(d, S)$ is the fuzzy soft set over $U$ and $(d, S)$ satisfies (3), we have

$$
R\left(R(f(s)(u), g(t)(v)), R\left(d(s)(u), g^{*}(t)(v)\right)\right) \geqslant \lambda .
$$

By Theorem 1(6), we have

$$
\begin{aligned}
& R\left(d(s)(u), R\left(\lambda, R\left(R(f(s)(u), g(t)(v)), g^{*}(t)(v)\right)\right)\right) \\
& =R\left(\lambda, R\left(d(s)(u), R\left(R(f(s)(u), g(t)(v)), g^{*}(t)(v)\right)\right)\right) \\
& =R\left(\lambda, R\left(R(f(s)(u), g(t)(v)), R\left(d(s)(u), g^{*}(t)(v)\right)\right)\right) \\
& =1 .
\end{aligned}
$$

Thus, we get conclude

$$
\begin{array}{r}
d(s)(u) \leqslant \wedge_{t \in T} \wedge \wedge_{v \in V}(R(\lambda, R(R(f(s)(u), \\
\left.\left.\left.g(t)(v)), g^{*}(t)(v)\right)\right)\right)=f_{\lambda}^{*}(s)(u) .
\end{array}
$$

Corollary 9. Suppose that the implication operator in FSMT is $R_{L}$. Then the calculation formula of $\lambda-$ Triple I inference of (5) is expressed as follows: for each $s \in S, u \in U$,

$$
\begin{gathered}
f_{\lambda}^{*}(s)(u)=\wedge \wedge_{t \in T} \wedge \hat{v \in V}\left(\left(1-\lambda+g^{*}(t)(v)+F(s)(u)\right.\right. \\
\left.-g(t)(v)) \wedge\left(1-\lambda+g^{*}(t)(v)\right) \wedge 1\right) .
\end{gathered}
$$

Corollary 10. Suppose that the implication operator in FSMT is $R_{G}$. Then the calculation formula of 
$\lambda$-Triple I inference of (5) is expressed as follows: for each $s \in S, u \in U$,

$$
\begin{gathered}
f_{\lambda}^{*}(s)(u)=\hat{}_{t \in T} \underset{v \in V}{\wedge} R_{G}\left(R_{G}(f(s)(u),\right. \\
\left.g(t)(v)), g^{*}(t)(v)\right),
\end{gathered}
$$

where $E(s, t, u)=\{v \in V ; R(R(f(s)(u), g(t)(v))$, $\left.\left.g^{*}(t)(v)\right)<\lambda\right\}$.

Remark 1. In $\lambda$-Triple I FSMP principle and $\lambda$-Triple I FSMT principle, if $\lambda=1$, we can get the computational formulas (1) and (2).

\section{Robustness of general Triple I fuzzy soft inference}

Before discussing the robustness analysis of Triple I fuzzy soft inference, we will introduce some necessary notions and theorems related to the content.

Now, assume that $F S(U, E)$ are the set of al1 fuzzy soft sets over $U$ and the implication operator related to left-continuous triangular norm $T$ is $R$.

Theorem 11. Suppose that $(f, S),(g, T)$ and $(k, L)$ are fuzzy soft sets over $U, V$ and $W$ respectively and $h_{i}:[0,1]^{3} \rightarrow[0,1], i=1,2,3,4$. For any $\lambda \in[0,1]$, $u \in U, s \in S, v \in V, t \in T, w \in W, l \in L$,

$$
\begin{aligned}
& \begin{array}{l}
h_{1}(f(s)(u), g(t)(v), k(l)(w)) \\
=
\end{array} \quad T(R(f(s)(u), g(t)(v)), k(l)(w)), \\
& h_{2}(f(s)(u), g(t)(v), k(l)(w)) \\
& =R(R(f(s)(u), g(t)(v)), k(l)(w)), \\
& h_{3}(f(s)(u), g(t)(v), k(l)(w)) \\
& =T(T(\lambda, R(f(s)(u), g(t)(v))), k(l)(w)), \\
& h_{4}(f(s)(u), g(t)(v), k(l)(w)) \\
& =R(\lambda, R(R(f(s)(u), g(t)(v)), k(l)(w))) .
\end{aligned}
$$

$$
\begin{gathered}
\text { If } f(s)(u) \leftrightarrow f^{\prime}(s)(u) \geqslant \delta_{1}, g(t)(v) \leftrightarrow \\
g^{\prime}(t)(v) \geqslant \delta_{2}, k(l)(w) \leftrightarrow k^{\prime}(l)(w) \geqslant \delta_{3}, \text { then for } \\
i=1,2,3,4, \\
h_{i}(f(s)(u), g(t)(v), k(l)(w)) \leftrightarrow h_{i}\left(f^{\prime}(s)(u),\right. \\
\left.g^{\prime}(t)(v), k^{\prime}(l)(w)\right) \geqslant T\left(\delta_{1}, \delta_{2}, \delta_{3}\right) .
\end{gathered}
$$

Proof. Let $\lambda=1$. By $T(1, x)=x, R(1, x)=x$, we have

$$
\begin{aligned}
& h_{3}(f(s)(u), g(t)(v), k(l)(w)) \\
& \quad=h_{1}(f(s)(u), g(t)(v), k(l)(w)), \\
& h_{4}(f(s)(u), g(t)(v), k(l)(w)) \\
& \quad=h_{2}(f(s)(u), g(t)(v), k(l)(w)) .
\end{aligned}
$$

So,we just proof the situations of $i=3,4$. For any $\lambda \in[0,1], u \in U, s \in S, v \in V, t \in T, w \in W, l \in L$,

$$
\begin{aligned}
& h_{3}(f(s)(u), g(t)(v), k(l)(w)) \leftrightarrow \\
h_{3}\left(f^{\prime}(s)(u), g^{\prime}(t)(v), k^{\prime}(l)(w)\right) & \\
= & T(T(\lambda, R(f(s)(u), g(t)(v))), k(l)(w)) \leftrightarrow \\
& T\left(T\left(\lambda, R\left(f^{\prime}(s)(u), g^{\prime}(t)(v)\right)\right), k^{\prime}(l)(w)\right) \\
\geqslant & T\left(T ( \lambda , R ( f ( s ) ( u ) , g ( t ) ( v ) ) ) \leftrightarrow T \left(\lambda, R\left(f^{\prime}(s)(u),\right.\right.\right. \\
& \left.\left.\left.g^{\prime}(t)(v)\right)\right), k(l)(w) \leftrightarrow k^{\prime}(l)(w)\right) \\
\geqslant & T(T(\lambda \leftrightarrow \lambda, R(f(s)(u), g(t)(v)) \leftrightarrow \\
& \left.\left.R\left(f^{\prime}(s)(u), g^{\prime}(t)(v)\right)\right), k(l)(w) \leftrightarrow k^{\prime}(l)(w)\right) \\
= & T\left(R(f(s)(u), g(t)(v)) \leftrightarrow R\left(f^{\prime}(s)(u), g^{\prime}(t)(v)\right),\right. \\
& \left.k(l)(w) \leftrightarrow k^{\prime}(l)(w)\right) \\
\geqslant & T\left(T \left(f(s)(u) \leftrightarrow f^{\prime}(s)(u), g(t)(v) \leftrightarrow\right.\right.
\end{aligned}
$$




$$
\begin{aligned}
&\left.\left.g^{\prime}(t)(v)\right), k(l)(w) \leftrightarrow k^{\prime}(l)(w)\right) \\
& \geqslant T\left(\delta_{1}, \delta_{2}, \delta_{3}\right) . \\
& h_{4}(f(s)(u), g(t)(v), k(l)(w)) \leftrightarrow \\
& h_{4}\left(f^{\prime}(s)(u), g^{\prime}(t)(v), k^{\prime}(l)(w)\right) \\
&= R(\lambda, R(R(f(s)(u), g(t)(v)), k(l)(w))) \leftrightarrow \\
& R\left(\lambda, R\left(R\left(f^{\prime}(s)(u), g^{\prime}(t)(v)\right), k^{\prime}(l)(w)\right)\right) \\
& \geqslant T(\lambda \leftrightarrow \lambda, R(R(f(s)(u), g(t)(v)), k(l)(w)) \\
&\left.\leftrightarrow R\left(R\left(f^{\prime}(s)(u), g^{\prime}(t)(v)\right), k^{\prime}(l)(w)\right)\right) \\
&= T\left(R(f(s)(u), g(t)(v)) \leftrightarrow R\left(f^{\prime}(s)(u), g^{\prime}(t)(v)\right),\right. \\
&\left.k(l)(w) \leftrightarrow k^{\prime}(l)(w)\right) \\
& \geqslant T\left(T \left(f(s)(u) \leftrightarrow f^{\prime}(s)(u), g(t)(v)\right.\right. \\
&\left.\left.\leftrightarrow g^{\prime}(t)(v)\right), k(l)(w) \leftrightarrow k^{\prime}(l)(w)\right) \\
&\left.\delta_{1}, \delta_{2}, \delta_{3}\right) .
\end{aligned}
$$

Cai presented $\delta$-equalities with respect to algebraic operators, fuzzy relations, FMP and $\mathrm{FMT}^{7,33}$. Georgescu proposed the so-called $(T, \delta)$-equality ${ }^{34}$. Now we will extend the notions to fuzzy soft sets. Assume that $(f, S),(g, T) \in$ $F S(U, E)$ and $R$ is fuzzy implication.

Definition 6. Assume that $\leftrightarrow$ is the biresidual associated with $R . w((f, S),(g, T))$ is similarity degree of $(f, S)$ and $(g, T)$, where

$$
w((f, S),(g, T))=\wedge_{s \in S \cap T} \wedge_{u \in U}(f(s)(u) \leftrightarrow g(s)(u)) .
$$

Definition 7. Assume that $0 \leqslant \delta \leqslant 1$. If $\sup _{\mathrm{u}, \mathrm{v} \in \mathrm{U}} \sup _{s, t \in S \cap T}|f(s)(u)-g(t)(v)| \leqslant 1-\delta$, we say that $(f, S)$ and $(g, T)$ are fuzzy soft $\delta$-equal, denoted $\operatorname{by}(f, S)={ }_{\delta}(g, T)$.

Definition 8. Assume that $T$ a left-continuous triangular norm and $0 \leqslant \delta \leqslant 1 .(f, S)$ and $(g, T)$ over $U$ are said to be $(T, \delta)$-equal for fuzzy soft sets, represented as $(f, S)=_{(T, \delta)}(g, T)$, if $w((f, S),(g, T)) \geqslant \delta$, where $w$ is the similarity degree associated with the residual of the t-norm $T$.

Now we study robustness analysis of Triple I fuzzy soft inference. We introduce some basic terminology and description: $R$ denotes the implication operators related to the left continuous triangular norm $T$, and $w$ denotes the similarity degree associated with $R, \rho$ denotes the biresidual related to $R$, $(f, S),\left(f^{*}, S\right)$ and $(g, T),\left(g^{*}, T\right)$ are fuzzy soft sets over $U$ and $V$ respectively.

Theorem 12. Assume that $w\left((f, S),\left(f^{\prime}, S\right)\right) \geqslant$ $\delta_{1}, w\left((g, T),\left(g^{\prime}, T\right)\right) \geqslant \delta_{2}, w\left(\left(f^{*}, S\right),\left(f^{*}, S\right)\right) \geqslant$ $\delta_{3}$, and $\left(g^{*}, T\right)$ and $\left(g^{*}, T\right)$ are Triple I solutions for $\operatorname{FSMP}\left((f, S),(g, T),\left(f^{*}, S\right)\right)$ and $\operatorname{FSMP}\left(\left(f^{\prime}, S\right)\right.$, $\left.\left(g^{\prime}, T\right),\left(f^{*}, S\right)\right)$ given by $(1)$ respectively. Then we have $w\left(\left(g^{*}, T\right),\left(g^{* *}, T\right)\right) \geqslant T\left(\delta_{1}, \delta_{2}, \delta_{3}\right)$.

Proof. By (1), we realize the solutions of Triple I inference method for FSMP are as follows: for any $v \in V, t \in T$,

$$
g^{\prime *}(t)(v)=\underset{s \in S}{\vee} \underset{u \in U}{\vee} T\left(R\left(f^{\prime}(s)(u), g^{\prime}(t)(v)\right), f^{\prime *}(s)(u)\right) .
$$

By Theorem 11, Lemma 2, Lemma 3 and Definition 6 , we have

$$
\begin{aligned}
& w\left(\left(g^{*}, T\right),\left(g^{*}, T\right)\right)=\bigwedge_{t \in T} \bigwedge_{v \in V} \rho\left(g^{*}(t)(v), g^{\prime *}(t)(v)\right) \\
& =\wedge_{t \in T} \bigwedge_{v \in V}\left(g^{*}(t)(v) \leftrightarrow g^{*}(t)(v)\right) \\
& =\wedge \hat{t \in T} \underset{v \in V}{\wedge}\left(\underset{s \in S u \in U}{\vee} T\left(R(f(s)(u), g(t)(v)), f^{*}(s)(u)\right)\right. \\
& \left.\leftrightarrow \underset{s \in S}{\vee} \underset{v \in U}{\vee} T\left(R\left(f^{\prime}(s)(u), g^{\prime}(t)(v)\right), f^{\prime *}(s)(u)\right)\right)
\end{aligned}
$$

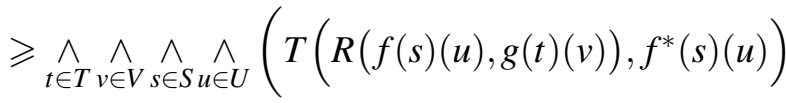

$$
\begin{aligned}
& \left.\leftrightarrow T\left(R\left(f^{\prime}(s)(u), g^{\prime}(t)(v)\right), f^{\prime *}(s)(u)\right)\right)
\end{aligned}
$$




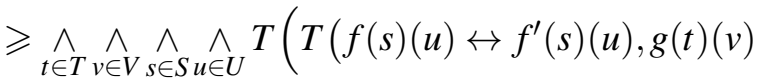

$$
\begin{aligned}
& \left.\left.\leftrightarrow g^{\prime}(t)(v)\right), f^{*}(s)(u) \leftrightarrow f^{\prime *}(s)(u)\right) \\
& \geqslant T\left(\delta_{1}, \delta_{2}, \delta_{3}\right) \text {. }
\end{aligned}
$$

Theorem 13. Assume that $w\left((f, S),\left(f^{\prime}, S\right)\right) \geqslant$ $\delta_{1}, w\left((g, T),\left(g^{\prime}, T\right)\right) \geqslant \delta_{2}, w\left(\left(g^{*}, T\right),\left(g^{\prime *}, T\right)\right) \geqslant$ $\delta_{3}$, and $\left(f^{*}, S\right)$ and $\left(f^{*}, S\right)$ are Triple I solutions of FSMT $\left((f, S),(g, T),\left(g^{*}, T\right)\right)$ and $F S M T\left(\left(f^{\prime}, S\right)\right.$, $\left.\left(g^{\prime}, T\right),\left(g^{\prime *}, T\right)\right)$ given by $(2)$ respectively. Then we have $w\left(\left(f^{*}, S\right),\left(f^{*}, S\right)\right) \geqslant T\left(\delta_{1}, \delta_{2}, \delta_{3}\right)$.

Proof. By (2), we realize the solutions of Triple I inference method for FSMT are as follows: for any $v \in V, t \in T$,

$f^{\prime *}(s)(u)=\wedge \hat{t \in T} \wedge \hat{v \in V}\left(R\left(R\left(f^{\prime}(s)(u), g^{\prime}(t)(v)\right), g^{\prime *}(t)(v)\right)\right)$.

By Theorem 11, Lemma 2, Lemma 3 and Definition 6 , we have

$$
\begin{aligned}
& w\left(\left(f^{*}, S\right),\left(f^{*}, S\right)\right)=\bigwedge_{s \in S} \bigwedge_{u \in U} \rho\left(f^{*}(s)(u), f^{\prime *}(s)(u)\right) \\
& =\wedge \wedge_{s \in S} \wedge f_{u}\left(f^{*}(s)(u) \leftrightarrow f^{*}(s)(u)\right) \\
& =\wedge \wedge_{s \in S} \hat{u \in U}\left(\hat{t \in T} \wedge \hat{v \in V}\left(R\left(R(f(s)(u), g(t)(v)), g^{*}(t)(v)\right)\right)\right. \\
& \left.\leftrightarrow \wedge \hat{t \in T} \wedge \in \wedge_{v \in V}\left(R\left(R\left(f^{\prime}(s)(u), g^{\prime}(t)(v)\right), g^{\prime *}(t)(v)\right)\right)\right) \\
& \geqslant \wedge \wedge \hat{s \in S} \wedge \wedge_{u \in T} \wedge \wedge_{v \in V}\left(R\left(R(f(s)(u), g(t)(v)), g^{*}(t)(v)\right)\right. \\
& \left.\leftrightarrow R\left(R\left(f^{\prime}(s)(u), g^{\prime}(t)(v)\right), g^{\prime *}(t)(v)\right)\right) \\
& \geqslant \wedge \wedge \wedge \wedge \hat{\wedge} \wedge \wedge_{s \in U} \wedge_{t \in V} T\left(T \left(f(s)(u) \leftrightarrow f^{\prime}(s)(u), g(t)(v)\right.\right. \\
& \left.\left.\leftrightarrow g^{\prime}(t)(v)\right), g^{*}(t)(v) \leftrightarrow g^{\prime *}(t)(v)\right)
\end{aligned}
$$$$
\geqslant T\left(\delta_{1}, \delta_{2}, \delta_{3}\right)
$$

Now, we generalize the above content, we have the following Theorems.

Theorem 14. Assume that $w\left((f, S),\left(f^{\prime}, S\right)\right) \geqslant \delta_{1}$, $w\left((g, T),\left(g^{\prime}, T\right)\right) \geqslant \delta_{2}, w\left(\left(f^{*}, S\right),\left(f^{\prime *}, S\right)\right) \geqslant \delta_{3}$, and $\left(g_{\lambda}{ }^{*}, T\right)$ and $\left(g_{\lambda}^{\prime}{ }^{*}, T\right)$ are $\lambda$-Triple I solutions of $\operatorname{FSMP}\left((f, S),(g, T),\left(f^{*}, S\right)\right)$ and $\operatorname{FSMT}\left(\left(f^{\prime}, S\right)\right.$, $\left.\left(g^{\prime}, T\right),\left(f^{\prime *}, S\right)\right)$ given by $(3)$ respectively. Then we $w\left(\left(g_{\lambda}{ }^{*}, T\right),\left(g_{\lambda}^{\prime}{ }^{*}, T\right)\right) \geqslant T\left(\delta_{1}, \delta_{2}, \delta_{3}\right)$.

Proof. By (3), we realize the solutions of $\lambda$-Triple I inference method for FSMP are as follows: for any $v \in V, t \in T$,

$$
\begin{aligned}
& g_{\lambda}{ }^{*}(t)(v)=\underset{s \in S}{\vee} \underset{u \in U}{\vee} T(T(\lambda, R(f(s)(u), \\
& \left.g(t)(v))), f^{*}(s)(u)\right) \\
& g_{\lambda}^{\prime *}(t)(v)=\underset{s \in S}{\vee} \underset{u \in U}{\vee} T\left(T \left(\lambda, R\left(f^{\prime}(s)(u),\right.\right.\right. \\
& \left.\left.\left.g^{\prime}(t)(v)\right)\right), f^{*}(s)(u)\right) .
\end{aligned}
$$

By Theorem 11, Lemma 2, Lemma 3 and Definition 6 , we have

$$
\begin{aligned}
& w\left(\left(g_{\lambda}{ }^{*}, T\right),\left(g_{\lambda}^{\prime}{ }^{*}, T\right)\right)=\wedge_{t \in T} \wedge_{v \in V} \rho\left(g_{\lambda}{ }^{*}(t)(v), g_{\lambda}^{\prime *}(t)(v)\right) \\
& =\wedge_{t \in T} \wedge \wedge_{v \in V}\left(g_{\lambda}^{*}(t)(v) \leftrightarrow g_{\lambda}^{\prime *}(t)(v)\right) \\
& =\wedge \wedge_{t \in T} \wedge{ }_{v \in V}(T(\lambda, \underset{s \in S}{\vee} \underset{u \in U}{\vee} T(R(f(s)(u), g(t)(v)), \\
& \left.\left.f^{*}(s)(u)\right)\right) \leftrightarrow T\left(\lambda, \underset{s \in S}{\vee} \underset{u \in U}{\vee} T\left(R \left(f^{\prime}(s)(u),\right.\right.\right. \\
& \left.\left.\left.\left.g^{\prime}(t)(v)\right), f^{\prime *}(s)(u)\right)\right)\right) \\
& =\wedge \wedge_{t \in T} \wedge\left(T\left(\lambda, g^{*}(t)(v)\right) \leftrightarrow T\left(\lambda, g^{*}(t)(v)\right)\right) \\
& \geqslant \wedge \wedge_{t \in T} \wedge \wedge_{v \in V} T\left(\lambda \leftrightarrow \lambda, g^{*}(t)(v) \leftrightarrow g^{*}(t)(v)\right) \\
& \geqslant \wedge \wedge_{t \in T} \wedge g_{v \in V}\left(g^{*}(t)(v) \leftrightarrow g^{* *}(t)(v)\right) \\
& \geqslant T\left(\delta_{1}, \delta_{2}, \delta_{3}\right) \text {. }
\end{aligned}
$$

Theorem 15. Assume that $w\left((f, S),\left(f^{\prime}, S\right)\right) \geqslant$ $\delta_{1}, w\left((g, T),\left(g^{\prime}, T\right)\right) \geqslant \delta_{2}, w\left(\left(g^{*}, T\right),\left(g^{\prime *}, T\right)\right) \geqslant \delta_{3}$, and $\left(f_{\lambda}{ }^{*}, S\right)$ and $\left(f_{\lambda}^{\prime *}, S\right)$ are $\lambda$-Triple I solution$s$ of FSMT( $\left.(f, S),(g, T),\left(g^{*}, T\right)\right)$ and $F S M T\left(\left(f^{\prime}, S\right)\right.$, $\left.\left(g^{\prime}, T\right),\left(g^{\prime *}, T\right)\right)$ given by (4) respectively. Then we 
have $w\left(\left(f_{\lambda}^{*}, S\right),\left(f_{\lambda}^{*}, S\right)\right) \geqslant T\left(\delta_{1}, \delta_{2}, \delta_{3}\right)$.

Proof. By (4), we realize the solutions of $\lambda$-Triple I inference method for FSMT are as follows: for any $u \in U, s \in S$,

$$
\begin{aligned}
& f_{\lambda}^{*}(s)(u)=\underset{t \in T}{\wedge} \wedge_{v \in V} R(\lambda, R(R(f(s)(u), g(t)(v)), \\
& \left.\left.g^{*}(t)(v)\right)\right) \text {, } \\
& f_{\lambda}^{\prime *}(s)(u)=\wedge_{t \in T} \wedge_{v \in V} R\left(\lambda, R\left(R\left(f^{\prime}(s)(u), g^{\prime}(t)(v)\right),\right.\right. \\
& \left.\left.g^{\prime *}(t)(v)\right)\right) \text {. }
\end{aligned}
$$

By Theorem 11, Lemma 2, Lemma 3 and Definition 6 , we have

$$
\begin{aligned}
& w\left(\left(f_{\lambda}^{*}, S\right),\left(f_{\lambda}^{*}, S\right)\right)=\bigwedge_{s \in S} \bigwedge_{u \in U} \rho\left(f_{\lambda}^{*}(s)(u), f_{\lambda}^{\prime *}(s)(u)\right) \\
& =\underset{s \in S u \in U}{\wedge}\left(f_{\lambda}^{*}(s)(u) \leftrightarrow f_{\lambda}^{\prime *}(s)(u)\right) \\
& =\wedge \wedge_{s \in S} \wedge\left(\wedge_{t \in T} \wedge_{v \in V} R\left(\lambda, R\left(R(f(s)(u), g(t)(v)), g^{*}(t)(v)\right)\right)\right. \\
& \left.\leftrightarrow \wedge_{t \in T} \wedge \wedge_{v \in V} R\left(\lambda, R\left(R\left(f^{\prime}(s)(u), g^{\prime}(t)(v)\right), g^{\prime *}(t)(v)\right)\right)\right) \\
& =\wedge \wedge_{s \in S} \wedge_{u \in U}\left(R\left(\lambda, f^{*}(s)(u)\right) \leftrightarrow R\left(\lambda, f^{\prime *}(s)(u)\right)\right)
\end{aligned}
$$

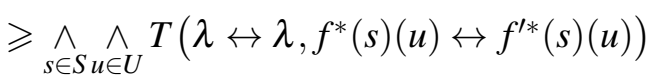

$$
\begin{aligned}
& \geqslant \wedge_{s \in S} \wedge \wedge_{u \in U}\left(f^{*}(s)(u) \leftrightarrow f^{*}(s)(u)\right) \\
& \geqslant T\left(\delta_{1}, \delta_{2}, \delta_{3}\right) \text {. }
\end{aligned}
$$

Remark 2. From the above theorems, we know that $w\left(\left(g_{\lambda}{ }^{*}, T\right),\left(g_{\lambda}^{\prime}{ }^{*}, T\right)\right)$ and $w\left(\left(f_{\lambda}{ }^{*}, S\right),\left(f_{\lambda}^{\prime *}, S\right)\right)$ are explicitly related to $\delta_{1}, \delta_{2}, \delta_{3}$ and $T$, is no explicit concern about $\lambda$.

\section{The Robustness of general Triple I method for multiple fuzzy soft rules for FSMP}

In the above content, we have considered general triple I method with respect to a single fuzzy soft rule. In practical problems, we usually need to discuss multiple fuzzy soft rules. The MFSMP is expressed as:

MFSMP: Major premise: $\left(f_{i}, S\right) \rightarrow\left(g_{i}, T\right)$ and Minor premise: $\left(f^{*}, S\right)$. Conclusion: $\left(g^{*}, T\right)$.

Where $i \in I, I=\{1, \cdots, n\}\left(f_{i}, S\right),\left(f^{*}, S\right)$ and $\left(g_{i}, T\right),\left(g^{*}, T\right)$ are fuzzy soft sets on $U$ and $V$ respectively. Now, we will consider how to extend the general triple I method to dispose multiple fuzzy soft rules and discuss their robustness.

In the same way, for given $\lambda \in[0,1]$, our purpose is to seek the optimal solution satisfying the following: The fuzzy soft set $\left(g^{*}, T\right)$ is the smallest fuzzy soft set on $V$ satisfing the following conditions:

(1)

$$
\begin{array}{r}
R\left(R\left(f_{i}(s)(u), g_{i}(t)(v)\right), R\left(f^{*}(s)(u),\right.\right. \\
\left.\left.g^{*}(t)(v)\right)\right) \geqslant \lambda, 1 \leqslant i \leqslant n
\end{array}
$$

(2)

$$
\text { where } r=\underset{i \in I}{\vee} R_{i}\left(\left(f_{i}, S\right),\left(g_{i}, T\right)\right) \text {. }
$$

In essence, the first case is first illation then polymerization. That is to say, by the $\mathrm{n}$ individual illations, we can get the $\mathrm{n}$ individual $\left(g_{\lambda_{i}}{ }^{*}, T\right)$ and polymerize these $\left(g_{\lambda_{i}}{ }^{*}, T\right)$ into the final conclusion. Hence, we have the following results:

Theorem 16. The smallest fuzzy soft set $\left(g_{\lambda}{ }^{*}, T\right)$ on $V$ satisfying the above condition(6) can be expressed as follows: for each $t \in T, v \in V$,

$$
\begin{gathered}
g_{\lambda}^{*}(t)(v)=\underset{i \in I}{\vee} \underset{s \in S}{\vee} \underset{u \in U}{\vee} T\left(T \left(\lambda, R\left(f_{i}(\alpha)(x),\right.\right.\right. \\
\left.\left.\left.g_{i}(t)(v)\right)\right), f^{*}(s)(u)\right)
\end{gathered}
$$

Proof. Firstly, we prove that $g_{\lambda}{ }^{*}(t)(v)$ defined by (8) satisfying (6). For each $s \in S, t \in T, u \in U, v \in V$, $i \in I$, we have

$g_{\lambda}{ }^{*}(t)(v) \geqslant T\left(T\left(\lambda, R\left(f_{i}(s)(u), g_{i}(t)(v)\right)\right), f^{*}(s)(u)\right)$

By Theorem 1(2), it follows that

$T\left(\lambda, R\left(f_{i}(s)(u), g_{i}(t)(v)\right)\right) \leqslant R\left(f^{*}(s)(u), g_{\lambda}{ }^{*}(t)(v)\right)$, 
$\lambda \leqslant R\left(R\left(f_{i}(s)(u), g_{i}(t)(v)\right), R\left(f^{*}(s)(u), g_{\lambda}^{*}(t)(v)\right)\right)$

Next, suppose that $(h, T)$ is the fuzzy soft set over $V$ and $(h, T)$ satisfies (6). Then we have

$$
R\left(R\left(f_{i}(s)(u), g_{i}(t)(v)\right), R\left(f^{*}(s)(u), h(t)(v)\right)\right) \geqslant \lambda
$$

for each $s \in S, t \in T, u \in U, v \in V, i \in I$.

By Theorem 1(5), it follows that

$$
\begin{aligned}
& R\left(T\left(T\left(\lambda, R\left(f_{i}(s)(u), g_{i}(t)(v)\right)\right), f^{*}(s)(u)\right), h(t)(v)\right) \\
& =R\left(T\left(\lambda, R\left(f_{i}(s)(u), g_{i}(t)(v)\right)\right), R\left(f^{*}(s)(u), h(t)(v)\right)\right) \\
& =R\left(\lambda, R\left(R\left(f_{i}(s)(u), g_{i}(t)(v)\right), R\left(f^{*}(s)(u), h(t)(v)\right)\right)\right) \\
& =1 .
\end{aligned}
$$

Thus, we can conclude that $T\left(T\left(\lambda, R\left(f_{i}(s)(u), g_{i}(t)(v)\right)\right)\right.$, $\left.f^{*}(s)(u)\right) \leqslant h(t)(v)$ and hence

$$
\begin{gathered}
g_{\lambda}^{*}(t)(v)=\underset{i \in I}{\vee} \underset{s \in S}{\vee} \underset{u \in U}{\vee} T\left(T\left(\lambda, R\left(f_{i}(s)(u), g_{i}(t)(v)\right)\right),\right. \\
\left.f^{*}(s)(u)\right) \leqslant h(t)(v) .
\end{gathered}
$$

Similarity, for the second case, we first polymerize every fuzzy soft rule $R\left(f_{i}(s)(u), g_{i}(t)(v)\right)$ into a single fuzzy soft relation $r$ and then get the conclusion by composing the $f^{*}(s)(u)$, that is to say, we get the conclusion for first polymerization then illation in the following:

Theorem 17. Assume that $r=\underset{i \in I}{\vee} R_{i}\left(\left(f_{i}, S\right),\left(g_{i}, T\right)\right)$.

Then the smallest on $V$ fuzzy soft set $\left(g_{\lambda}{ }^{*}, T\right)$ satisfying the above condition (7) can be expressed as follows: for each $t \in T, v \in V$,

$$
g_{\lambda}^{*}(t)(v)=\underset{s \in S}{\vee} \underset{u \in U}{\vee} T\left(T(\lambda, r), f^{*}(s)(u)\right)
$$

Proof. Firstly, we prove that $g_{\lambda}{ }^{*}(t)(v)$ defined by (9) satisfying (7). For each $s \in S, t \in T, u \in U, v \in V$, $i \in I, I=1, \cdots, n$ we have

$$
g_{\lambda}{ }^{*}(t)(v) \geqslant \underset{s \in S}{\vee} \underset{u \in U}{\vee} T\left(T(\lambda, r), f^{*}(s)(u)\right)
$$

By Theorem 1(2), it follows that

$$
\begin{aligned}
& T(\lambda, r) \leqslant R\left(f^{*}(s)(u), g_{\lambda}^{*}(t)(v)\right), \\
& R\left(r, R\left(f^{*}(s)(u), g_{\lambda}^{*}(t)(v)\right)\right) \geqslant \lambda .
\end{aligned}
$$

Next, suppose that $(h, T)$ is the fuzzy soft set over $V$ and $(h, T)$ satisfies (7). Then we have

$$
R\left(r, R\left(f^{*}(s)(u), h(t)(v)\right)\right) \geqslant \lambda .
$$

for each $s \in S, t \in T, u \in U, v \in V$.

By Theorem 1(2), it follows that

$$
\begin{aligned}
& T(\lambda, r) \leqslant R\left(f^{*}(s)(u), h(t)(v)\right), \\
& T\left(T(\lambda, r), f^{*}(s)(u)\right) \leqslant h(t)(v) .
\end{aligned}
$$

Thus, we can conclude that

$$
g_{\lambda}{ }^{*}(t)(v)=\underset{s \in S}{\vee} \underset{u \in U}{\vee} T\left(T(\lambda, r), f^{*}(s)(u)\right) \leqslant h(t)(v)
$$

Remark 3. If $i=1$ in the above Theorem 16 and Theorem 17, we can get Theorem 2 .

Now, we consider analysing the robustness of general Triple I inference for multiple fuzzy soft rules for FSMP.

Theorem 18. Assume that $w\left(\left(f_{i}, S\right),\left(f_{i}^{\prime}, S\right)\right) \geqslant \delta_{1}$, $w\left(\left(g_{i}, T\right),\left(g_{i}^{\prime}, T\right)\right) \geqslant \delta_{2}, w\left(\left(f^{*}, S\right),\left(f^{*}, S\right)\right) \geqslant \delta_{3}$, and $\left(g_{\lambda}{ }^{*}, T\right)$ and $\left(g_{\lambda}^{\prime}{ }^{*}, T\right)$ are general Triple I solutions for multiple fuzzy soft rules given by (8) respectively. Then we have $w\left(\left(g_{\lambda}{ }^{*}, T\right),\left(g_{\lambda}^{\prime}{ }^{*}, T\right)\right) \geqslant$ $T\left(\delta_{1}, \delta_{2}, \delta_{3}\right)$.

Proof. By (8), we realize the solutions of general Triple I inference method for multiple fuzzy soft rules are as follows: for each $t \in T, v \in V$,

$$
\begin{gathered}
g_{\lambda}^{*}(t)(v)=\underset{i \in I \in S \in S u \in U}{\vee} \underset{s}{\vee} T\left(T \left(\lambda, R\left(f_{i}(s)(u),\right.\right.\right. \\
\left.\left.\left.g_{i}(t)(v)\right)\right), f^{*}(s)(u)\right)
\end{gathered}
$$

By Lemma 2, Lemma 3 and Definition 6, we have 


$$
\begin{aligned}
& w\left(\left(g_{\lambda}{ }^{*}, T\right),\left(g_{\lambda}^{\prime *}, T\right)\right)=\wedge_{t \in T} \wedge_{v \in V} \rho\left(g_{\lambda}{ }^{*}(t)(v), g_{\lambda}^{\prime *}(t)(v)\right) \\
& =\wedge \wedge_{t \in T} \wedge{ }_{v \in V}\left(g_{\lambda}^{*}(t)(v) \leftrightarrow g_{\lambda}^{\prime *}(t)(v)\right) \\
& =\wedge \wedge_{t \in T} \wedge \underset{v \in V}{\wedge} \underset{i \in I}{\vee} \underset{s \in S}{\vee} \underset{u \in U}{\vee} T\left(T\left(\lambda, R\left(f_{i}(s)(u), g_{i}(t)(v)\right)\right),\right. \\
& \left.f^{*}(s)(u)\right) \leftrightarrow \underset{i \in I}{\vee} \underset{s \in S}{\vee} \underset{u \in U}{\vee} T\left(T \left(\lambda, R\left(f_{i}^{\prime}(s)(u),\right.\right.\right. \\
& \left.\left.\left.\left.g_{i}^{\prime}(t)(v)\right)\right), f^{\prime *}(s)(u)\right)\right)
\end{aligned}
$$

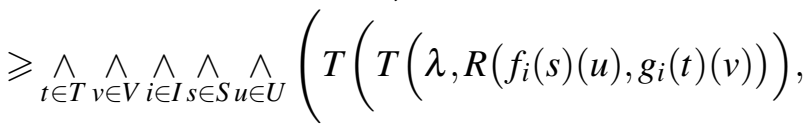

$$
\begin{aligned}
& \left.f^{*}(s)(u)\right) \leftrightarrow T\left(T\left(\lambda, R\left(f_{i}^{\prime}(s)(u), g_{i}^{\prime}(t)(v)\right)\right),\right. \\
& \left.\left.f^{\prime *}(s)(u)\right)\right)
\end{aligned}
$$

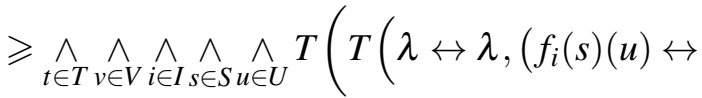

$$
\begin{aligned}
& \left.\left.g_{i}(t)(v)\right) \leftrightarrow\left(f_{i}^{\prime}(s)(u) \leftrightarrow g_{i}^{\prime}(t)(v)\right)\right), f^{*}(s)(u) \\
& \left.\leftrightarrow f^{\prime *}(s)(u)\right) \\
& \geqslant \hat{t \in T} \wedge \hat{v \in V} \wedge \hat{\wedge} \wedge \wedge_{i \in S} \wedge_{u \in U} T\left(T \left(f_{i}(s)(u) \leftrightarrow f_{i}^{\prime}(s)(u),\right.\right. \\
& \left.\left.g_{i}(t)(v) \leftrightarrow g_{i}^{\prime}(t)(v)\right), f^{*}(s)(u) \leftrightarrow f^{\prime *}(s)(u)\right) \\
& \geqslant T\left(\delta_{1}, \delta_{2}, \delta_{3}\right) \text {. }
\end{aligned}
$$

Similarity, we have the following conclusion for the second case:

Theorem 19. Assume that $w\left(\left(f_{i}, S\right),\left(f_{i}^{\prime}, S\right)\right) \geqslant$ $\delta_{1}, \quad w\left(\left(g_{i}, T\right),\left(g_{i}^{\prime}, T\right)\right) \geqslant \delta_{2}, w\left(\left(f^{*}, S\right),\left(f^{\prime *}, S\right)\right) \geqslant$ $\delta_{3}$, and $\left(g_{\lambda}{ }^{*}, T\right)$ and $\left(g_{\lambda}^{\prime}{ }^{*}, T\right)$ are general Triple I solutions for multiple fuzzy soft rules given by (9) respectively, where $r=\underset{i \in I}{\vee} R_{i}\left(\left(f_{i}, S\right),\left(g_{i}, T\right)\right)$ and $r^{\prime}=\underset{i \in I}{\vee} R_{i}^{\prime}\left(\left(f_{i}^{\prime}, S\right),\left(g_{i}^{\prime}, T\right)\right)$. Then we have $w\left(\left(g_{\lambda}{ }^{*}, T\right),\left(g_{\lambda}^{\prime *}, T\right)\right) \geqslant T\left(\delta_{1}, \delta_{2}, \delta_{3}\right)$.

Proof. By (9), Lemma 2, Lemma 3 and Definition 6 , we have

$$
\begin{aligned}
& w\left(\left(g_{\lambda}{ }^{*}, T\right),\left(g_{\lambda}^{\prime *}, T\right)\right)=\bigwedge_{t \in T} \bigwedge_{v \in V} \rho\left(g_{\lambda}{ }^{*}(t)(v), g_{\lambda}^{\prime *}(t)(v)\right) \\
& =\wedge_{t \in T} \wedge \wedge_{v \in V}\left(g_{\lambda}^{*}(t)(v) \leftrightarrow g_{\lambda}^{\prime *}(t)(v)\right) \\
& =\wedge \hat{t \in T} \underset{v \in V}{\wedge}\left(\underset{s \in S}{\vee} \underset{u \in U}{\vee} T\left(T(\lambda, r), f^{*}(s)(u)\right) \leftrightarrow \underset{s \in S}{\vee} \underset{u \in U}{\vee}\right. \\
& \left.T\left(T\left(\lambda, r^{\prime}\right), f^{\prime *}(s)(u)\right)\right) \\
& \geqslant \wedge \hat{t \in T} \wedge \hat{v} \wedge \wedge_{s \in S} \wedge \wedge_{u \in U}\left(T\left(T(\lambda, r), f^{*}(s)(u)\right) \leftrightarrow\right. \\
& \left.T\left(T\left(\lambda, r^{\prime}\right), f^{\prime *}(s)(u)\right)\right) \\
& \geqslant \wedge \hat{t \in T} \wedge \wedge_{v \in V} \wedge \wedge_{s \in S} \wedge_{u \in U} T\left(T\left(\lambda \leftrightarrow \lambda, r \leftrightarrow r^{\prime}\right), f^{*}(s)(u) \leftrightarrow\right. \\
& \left.f^{* *}(s)(u)\right) \\
& =\wedge \hat{t \in T} \wedge \hat{v \in V} \wedge \hat{s \in S} \wedge \wedge_{u \in U} T\left(T \left(\lambda \leftrightarrow \lambda, \underset{i \in I}{\vee} R_{i}\left(\left(f_{i}, S\right),\left(g_{i}, T\right)\right)\right.\right. \\
& \left.\leftrightarrow \underset{i \in I}{\vee} R_{i}{ }^{\prime}\left(\left(f_{i}{ }^{\prime}, S\right),\left(g_{i}{ }^{\prime}, T\right)\right)\right), f^{*}(s)(u) \\
& \left.\leftrightarrow f^{*}(s)(u)\right) \\
& \geqslant \wedge \wedge \hat{t \in T} \wedge \underset{v \in V}{\wedge} \wedge \wedge_{s \in S} \wedge \wedge_{u \in U} T\left(T \left(f_{i}(s)(u) \leftrightarrow f_{i}^{\prime}(s)(u),\right.\right. \\
& \left.\left.g_{i}(t)(v) \leftrightarrow g_{i}^{\prime}(t)(v)\right), f^{*}(s)(u) \leftrightarrow f^{\prime *}(s)(u)\right) \\
& \geqslant T\left(\delta_{1}, \delta_{2}, \delta_{3}\right)
\end{aligned}
$$

\section{Conclusions and Discussion}

In this paper, we investigated robustness of general Triple I method for fuzzy soft inference with respect to FSMP and FSMT models. We proved that the perturbation parameters of the general Triple I method for fuzzy soft inference are the same in the FSMP and FSMT models. The disturbance parameters do not change through $\lambda$. Finally, we discussed robustness of general triple I inference method for multiple fuzzy soft rule in the FSMP model. In the future work, we can consider a symmetric inference method based on the Triple I inference method for fuzzy soft sets and discuss the robustness of the soft symmetric inference. 


\section{Acknowledgments}

This work has been partially supported by the National Natural Science Foundation of China (Grant No. 61473239, 61372187, 61673320).

\section{References}

1. L.A Zadeh, Outline of a new approach to the analysis of complex systems and decision processes, IEEE Trans.Systems Man Cyber net (3)(1973)28-44.

2. D.G.Wang, Y.P.Meng, H.X.Li, A fuzzy similarity inference method for fuzzy reasoning, Computers and Mathematics with Applications 56(2008)2445-2454.

3. G.J. Wang, The full implicational triple I method for fuzzy reasoning, Science in China 29(1999)43-53.

4. S.J. Song, J. J. Yang, L. Zhang, Peter B. Luh, Reverse triple I method with restriction degree in fuzzy reasoning, Proceedings of the 4 World Congress on Intelligent Control and Automation June 1(14)(2002)15441548.

5. C.P. Pappis, Value approximation of fuzzy systems variables, Fuzzy Sets Syst 39(1991)111-115.

6. S. Dai, D. Pei, S.M. Wang, Perturbation of fuzzy sets and fuzzy reasoning based on normalized Minkowski distances, Fuzzy Sets and Systems 189(2012)63-73.

7. K.Y. Cai, Robustness of fuzzy reasoning and $\delta$-equalities of fuzzy sets, IEEE Transactions on Fuzzy Systems 9(5)(2001) 738-750.

8. Y.F. Li, K.Y. Qin, X.X. He, Robustness of fuzzy connectives and fuzzy reasoning, Fuzzy Sets and Systems 225 (2013)93C105.

9. Y.F. Li, K.Y. Qin, X.X. He, D. Meng, Robustness of fuzzy connectives and fuzzy reasoning with respect to general divergence measures, Fuzzy Sets and Systems 294(2016)63C78.

10. D. Molodtsov, The theory of soft sets, URSS Publisher, Moscow(in Russian) 2004.

11. D. Molodtsov, Soft set theory-first results,Computers and Mathematics with Applications 37(1999)19-31.

12. J.M. Zhan, M.I. Ali, N. Mehmood, On a novel uncertain soft set model: Z-soft fuzzy rough set model and corresponding decision making methods, Applied Soft Computing 56(2017)446-457.

13. J.M. Zhan, Q. Liu, W. Zhu, Another approach to rough soft hemirings and corresponding decision making Soft Computing 21(2017)3769-3780.

14. Y.Y. Liu, L. Martłnez and K.Y. Qin, A Comparative Study of Some Soft Rough Sets, Symmetry 11(9)(2017)252.

15. P.K. Maji, R.Biswas, A.R.Roy, Soft set theory, Computers and Mathematics with Applications 45(2003)555-562.

16. M.I. Ali, F. Feng, X. Liu, W.K. Min, M.Shabir, On some new operations in soft set theory, Computers and Mathematics with Applications 57(2009)1547-1553.

17. P.K. Maji, R.Biswas, A.R.Roy, Fuzzy soft sets, The Journal of Fuzzy Mathematics 9(3)(2001)589-602.

18. M. Khan, Reprsentation of intuitionistic fuzzy soft set using complex number, Journal of Applied Mathematics and Informatics 2017(35)331-347.

19. S. Bouznad, Generalized fuzzy soft set based fusion strategy for activity classification in smart home,IEEE International Conference on Fuzzy Systems 23,2017.

20. K.Y. Qin, J.L. Yang, Z.C. Liu. A fuzzy soft set based approximate reasoning method, Journal of Intelligentvand Fuzzy Systems (32)(2017)831-839.

21. L. Wang, B.B. Xue, K.Y. Qin, A similarity-based fuzzy soft reasoning method, The 2017 International Conference on Intelligent Systems and Knowledge Engineering (ISKE 2017) Nov. 24-26, Naijing.

22. B.B. Xue, L. Wang, K.Y. Qin, An interval-valued fuzzy soft set based Triple I method, The 2017 International Conference on Intelligent Systems and Knowledge Engineering (ISKE 2017) Nov. 24-26, Naijing.

23. B.B. Xue, K.Y. Qin, Properties of fuzzy soft set based triple I reasoning method, Computer science (5)2018.

24. J.H. Jin, Y.M. Li, C.Q. Li, Robustness of fuzzy reasoning via logically equivalence measure, Information Sciences 177(22)(2007)5103-5117.

25. M.X. Luo, k. Zhang, Robustness of full implication algorithms based on interval-valued fuzzy inference, International Journal of Approximate Reasoning 62(2015)61-72.

26. R. Zanotelli, R. Reiser, A. Yamin, B. Bedregal, Robustness of intuitionistc fuzzy difference operators, Uncertainty Modelling in knowledge Engineering and Decision-making Proceedings of the 12th International FLINS Conference FLINS 2016 37-43.

27. M. Baczynski, B. Jayaram, Fuzzy Implications, Fuzziness and Soft Computing Springer, Berlin Heidelberg, 2008(231).

28. E.P. Klement, R. Mesiar, E. Pap, Triangular norms, $K$ luwer Academic Publishers. Dordrecht 2000.

29. P. Hjek, Metamathematics of fuzzy Logic, Kluwer Academic Publishers, Dordrecht 1998.

30. G.J. Wang, Non-classical mathematical logic and approximate reasoning, Science Press, (Beijing, Chinese) 2000.

31. P. Kukkurainen, E. Turunen, Many-valued similarity reasoning. An Axiomatic Approach, Int. J. Multiple Valued Logic 8(2002)751C760.

32. E. Turunen, Mathematics behind fuzzy logic, PhysicaVerlag, Wurzburg 1999.

33. K.Y. Cai, $\delta$-equalities of fuzzy sets, Fuzzy Sets Syst 76(1995)97-112.

34. I. Georgescu, $(\delta, H)$-equality of Fuzzy Sets, MultiValued Logic Soft Comput 14(2008)1-32. 